\title{
Properties and Evolution of Emission in Molecular Aggregates of a Perylene Ammonium Derivative in Polymer Matrices
}

\author{
Fuyuki Ito, ${ }^{* a}$ Hirofumi Sato, ${ }^{a}$ Yuri Ugachi, ${ }^{a}$ Narumi Oka, ${ }^{a}$ Syoji Ito ${ }^{b}$ and Hiroshi Miyasaka ${ }^{b}$ \\ Received (in $X X X, X X X)$ Xth $X X X X X X X X X 20 X X$, Accepted Xth $X X X X X X X X X 20 X X$ \\ ${ }_{5}$ DOI: 10.1039/b000000x
}

\begin{abstract}
Size-dependent fluorescent properties of aggregates of a perylene ammonium derivative (PeryAm) were studied by steady-state and time-resolved spectroscopic methods. Quantitative analyses of aggregated states in aqueous solution indicated that the aggregation proceeded through dimer units of PeryAm. Fluorescence of the aggregate in the PVA film prepared from the aqueous solution continuously 10 redshifted with an increase in the concentration of PeryAm in the mother liquor, while keeping the absorption spectra almost the same band shapes. Fluorescence anisotropy values of aggregates in the PVA film were dependent on the monitoring wavelength and time profiles of the fluorescence in longer wavelength showed fast rise just after the pulsed excitation. These results indicated the efficient energy transfer to the stable sites in aggregates. Fluorescence microscope images showed aggregates were 15 segregated in the PVA film and the color of the emission was dependent on the size of the aggregate. Under the steady-state irradiation, the emission color of the aggregates was changed from green to yellow, which was attributable to the association of a small cluster of PeryAm with the green emission resulting in the formation of yellow-colored aggregates. On the basis of these results, we discussed the mechanisms and the dynamics of the aggregation and size-dependent emission in aggregates.
\end{abstract}

\section{${ }_{20}$ Introduction}

Organic molecular aggregates take an important role in various photofunctional systems. ${ }^{1,2}$ Fluorescence property of organic molecules in aggregates is different from those molecularly dissolved in solutions and various emission colors appear through 25 the molecular interaction depending on the assembled states. ${ }^{3,4}$ So as to control these emissive properties, it is of crucial importance to obtain the detailed information on the origin and evolution of the emission in the aggregated state where the specific interaction can lead to the appearance of new colors. ${ }^{5-10}$

30 Along this line, we studied fluorescence properties of aggregates in poly(vinyl alcohol) (PVA) matrix. ${ }^{11,} 12$ The segregation of solute molecules in PVA films prepares aggregates with a size of $\mathrm{nm}$ to $\mu \mathrm{m}$ and the high viscosity in the polymer film decelerates the temporal evolution of size and morphology 35 of aggregates. ${ }^{13,14}$ By applying this method of polymer matrix isolation, we found that the unique fluorescence in longer wavelength range appeared in the segregation processes of pyrene ammonium $(\mathrm{PyAm})^{11}$ and perylene ammonium (PeryAm) ${ }^{12}$ derivatives and, interestingly, this new emission continuously 40 redshifted with increasing concentration of the solute.

This gradual change of the fluorescence can provide the information on the aggregation and segregation processes through the evolution of the emissive species in the aggregates ${ }^{15}$ and suggested the possibility for the control of the emission color by 45 the control of the size and morphology of the aggregate. So as to precisely elucidate the mechanism on the evolution of the aggregate and obtain the rational principle for the control of the emission, comprehensive information on the dynamics of the aggregation is indispensable. Along this line, we have undertaken 50 concentration-dependent fluorescence change of anisotropy and fluorescence lifetime measurements of PeryAm in PVA matrices as well as the quantitative analyses of the aggregation in aqueous solution. Moreover, the fluorescence microscopy was applied to the detection of the aggregation and segregation processes in the ${ }_{55}$ present work. By integrating the continuous evolution of the emission properties of PeryAm in PVA films with increasing concentration, we discuss the evolution of the emissive state in the aggregates and the photoinduced switching of the emission of the aggregate in the following.

\section{${ }_{60}$ Experimental}

Perylene ammonium derivative (trimethyl-(2-oxo-3-perylene-1yl-ethyl)-ammonium bromide; PeryAm Fig. 1) was synthesized according to previous report. ${ }^{12}$ PVA (Tokyo Chemical Industry, $n$ $=1750 \pm 50$, degrees of saponification 97-100 mol\%) was used ${ }_{65}$ without further purification. PVA thin films doped with PeryAm were prepared by drop-casting onto quartz plates. The PVA concentration in ion-exchanged and distilled water was fixed to $0.113 \mathrm{~mol} \cdot \mathrm{dm}^{-3}(0.5 \mathrm{wt} \%)$. The coated films were dried under vacuum condition for $24 \mathrm{~h}$ at $298 \mathrm{~K}$. UV-Vis absorption spectra 70 were measured by a Shimadzu UV-2450 spectrometer. Fluorescence spectra were recorded by a Shimadzu RF-5300PC 
fluorescence spectrophotometer. Anisotropy of steady-state fluorescence was obtained by changing the polarization of the fluorescence either parallel or perpendicular to that of the excitation light. The anisotropy was calculated by using $r=\left(I_{\mathrm{VV}^{-}}\right.$ $\left.{ }_{5} G I_{\mathrm{VH}}\right) /\left(I_{\mathrm{VV}}+2 G I_{\mathrm{VH}}\right)$, where $I_{\mathrm{VV}}$ and $I_{\mathrm{VH}}$ respectively are the fluorescence intensity of the vertically polarized portion of fluorescence and that for the horizontally polarized portion under the excitation with the vertically polarized light. That is, the first and second subscripts represent excitation and detection ${ }_{10}$ polarization, respectively. ${ }^{16}$ The factor $G$ is defined by $I_{\mathrm{VV}} / I_{\mathrm{VH}}$, which is equal to the ratio of the sensitivities of the detection system for vertically and horizontally polarized light.

Fluorescence lifetime was measured by a time-correlated single-photon-counting (TCSPC) system. Experimental setup for ${ }_{15}$ the TCSPC was described previously. ${ }^{17}$ The excitation light source was the second harmonic $(400 \mathrm{~nm})$ of a Ti:sapphire laser (Spectra Physics, Tsunami). The fluorescence lifetime was estimated by non-liner least square fitting with deconvolution of the actual instrumental response function (IRF). Typical time 20 resolution by this method is ca. $5-10 \mathrm{ps}$.

The fluorescence color and the morphology of the films were captured by using an inverted microscope (Olympus IX71), equipped with a high-pressure mercury lamp, a dichroic mirror (Olympus WU filter cube), a UPlanF1 10×/0.3 (Olympus) 25 objective lens, a CCD camera (Sigma Koki SK-TC202USB-AT), and a USB 4000 (Ocean Optics) spectrometer which was not corrected for the wavelength dependence. ${ }^{15}$ The photoirradiation of the films under the microscope was also performed with the same high-pressure mercury lamp, a dichroic mirror (Olympus ${ }_{30}$ WU filter cube; excitation: 330-385 nm, monitor: over $420 \mathrm{~nm}$ ), the power of which was about $0.6 \mathrm{~mW}$. All experiments were carried out at room temperature $(298 \mathrm{~K})$.<smiles>C[N+](C)(C)CC(=O)c1ccc2c3cccc4cccc(c5cccc1c53)c42</smiles>

Fig. 1 Molecular structure of PeryAm.

\section{Results and Discussion}

\section{Concentration-dependent properties of PeryAm in aqueous} solution

Before the discussion on the concentration effect of PeryAm in 40 PVA film system, we show the concentration-dependent properties of PeryAm in aqueous solution as a reference. Parts of the results were described in the previous letter. ${ }^{12}$ Fig. 2a shows the absorption spectra of PeryAm in aqueous solution as a function of the concentration. New absorption band around 450 $45 \mathrm{~nm}$, which is different from that of the monomer, increases with increasing concentration. Spectral features such as absorption maximum and bandwidth were independent of the concentration.
In addition, the emission maximum and bandwidth of this new species were also independent of the concentration. ${ }^{21}$ These 50 results indicate that this new absorption is safely ascribable to only one species in this range of the concentration and suggest the formation of the dimer.

In order to quantitatively analyze this new absorption band, we assumed that the monomer-dimer equilibrium is established in the ${ }_{55}$ aqueous solution as shown in eq. (1).

$$
\mathrm{M}+\mathrm{M} \rightleftarrows \mathrm{D}
$$

The equilibrium constant $K$ can be represented as,

$$
{ }_{60} \quad K=\frac{[\mathrm{D}]}{[\mathrm{M}][\mathrm{M}]}=\frac{x / 2}{\left(\mathrm{M}_{0}-x\right)\left(\mathrm{M}_{0}-x\right)}
$$

where $\mathrm{M}_{0}$ is the total concentration of PeryAm in the solution and $x / 2$ corresponds to that of the dimer, [D]. In the case that $\mathrm{M}_{0}$ is much smaller than $1 / K$, the dimer concentration, [D], is ${ }_{65}$ quadratically proportional to $\mathrm{M}_{0}$. On the other hand, the dimer concentration is proportional to $\mathrm{M}_{0}$ in the case where $\mathrm{M}_{0}$ is much larger than $1 / K$. In the range with $\mathrm{M}_{0} \approx 1 / K$, the concentration of the dimer is expected to show the relation not being dependent on [D] $\propto \mathrm{M}_{0^{a}}(\alpha=1$ or 2$)$ (see details in ESI). Accordingly, the 70 analysis of the absorbance of the new species as a function of $\mathrm{M}_{0}$ in this intermediate range may provide detailed information of the equilibrium constant.

The dependence of the absorbance at $450 \mathrm{~nm}$ on $\mathrm{M}_{0}$ is shown in Fig. 2b, where both axes are given in logarithmic scales. In this 75 range, the absorbance of the new species can be approximated to be in proportion with $\mathrm{M}_{0}{ }^{a}$ with $\alpha=1.3$, indicating that the relation, $\mathrm{M}_{0} \approx 1 / K$, holds in this range. The solid line is a curve calculated using eq. (2) with $K=4.2 \times 10^{4} \mathrm{M}^{-1}$ and the molar absorption coefficient of the dimer as $44,800 \mathrm{M}^{-1} \mathrm{~cm}^{-1}$. The calculated curve ${ }_{80}$ in Fig. $2 \mathrm{~b}$ well reproduces the experimental results, indicating that the new absorption band is due to the formation of the dimer of PeryAm with the large equilibrium constant.

As mentioned above and in the previous letter ${ }^{12}$, this new species shows the fluorescence at $610 \mathrm{~nm}$. To confirm the 85 validity of the molar absorption coefficient of the dimer as estimated above, we evaluated the radiative rate constant, $k_{\mathrm{f}}$, of the dimer fluorescence using the modified Strickler-Berg relation. ${ }^{18}$ Fluorescence quantum yield $(\Phi)$ and the fluorescence lifetime $(\tau)$ of the dimer at the concentration of $1.0 \times 10^{-4} \mathrm{~mol} \cdot \mathrm{dm}^{-3}$ 90 were respectively obtained to be 0.22 and $360 \mathrm{ps}$, respectively (see ESI). From these values of the fluorescence properties, the $k_{\mathrm{f}}$ value was obtained to be $6.0 \times 10^{8} \mathrm{~s}^{-1}$. On the other hand, the radiative rate constant was estimated to be $4.6 \times 10^{8} \mathrm{~s}^{-1}$ using the modified Strickler-Berg relation with the molar absorption 95 coefficient of $44,800 \mathrm{M}^{-1} \mathrm{~cm}^{-1}$. These two values were comparable with each other and this result also supports the formation of the dimer in the aqueous solution.

Because the association constant of PeryAm in water is very large and the solubility in other solvent is very low, it was 100 difficult to purely obtain the absorption spectrum of the monomer of PeryAm. Hence, the excitation spectrum for the fluorescence at $470 \mathrm{~nm}$ was measured in the aqueous solution of PeryAm $\left(2.0 \times 10^{-6} \mathrm{~mol} \cdot \mathrm{dm}^{-3}\right)$ as shown in ESI. This spectrum showed three 
vibrational peaks at $392,414,440 \mathrm{~nm}$ and was in the mirror image of the fluorescence spectrum with peaks at 460 and 490 nm in Fig. 3b.
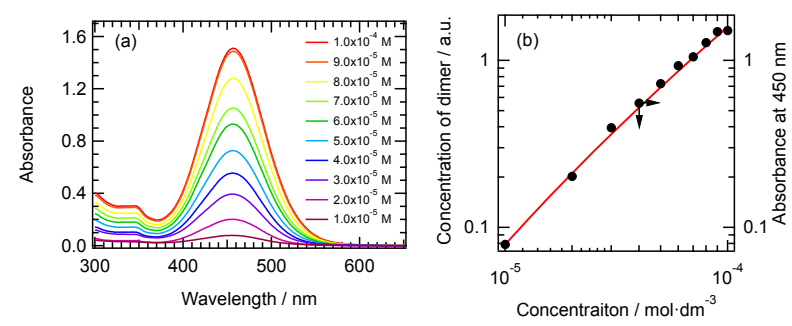

${ }_{5}$ Fig. 2 (a) Absorption spectra of PeryAm in aqueous solution. (b) The dependence of absorbance at $450 \mathrm{~nm}$ on the concentration of PeryAm in aqueous solution (closed circles). The solid line is a curve calculated using eq. (2) with the monomer-dimer equilibrium constant of $K=$ $4.2 \times 10^{4} \mathrm{M}^{-1}$. (see text)

10 Fig. 3 shows absorption and fluorescence spectra of PeryAm in PVA films as a function of concentration. With increasing concentration, new absorption band around $465 \mathrm{~nm}$ increases. The absorption maximum and the bandwidth were almost independent of the concentration. Because the absorption 15 maximum and the spectral band shape are similar to those in the aqueous solution in Fig. 2, this new band is ascribed to the dimer.

Fig. $3 \mathrm{~b}$ shows fluorescence spectra of PeryAm in PVA thin films with concentration from 0.001 to $0.4 \mathrm{~mol} \%$, excited at 400 $\mathrm{nm}$. Part of the results was reported in the previous letter. ${ }^{12}$

20 Although the absorption spectral shape was almost independent of the concentration as shown in Fig. 3a, the fluorescence spectra of PeryAm in PVA films were strongly dependent on the concentration. At the concentration of $0.001 \mathrm{~mol} \%$, the fluorescence peak was observed around $450 \mathrm{~nm}$. This ${ }_{25}$ fluorescence can be safely ascribed to the monomer on the basis of the fluorescence spectrum in the aqueous solution with diluted concentration. At $0.01 \mathrm{~mol} \%$, the intensity at $450 \mathrm{~nm}$ decreases and new broad band appears around $530 \mathrm{~nm}$. With further increase in the concentration of PeryAm, the band at $450 \mathrm{~nm}$ 30 disappears entirely and the broad emission appearing in longer wavelength region redshifts with increasing concentration of PeryAm. The fluorescence peak reached at $565 \mathrm{~nm}$ under the concentration of $0.4 \mathrm{~mol} \%$. This broad emission shifting toward longer wavelength with an increase in the concentration is 35 ascribable to the dimer in the PVA film. It is, however, worth noting that the emission maximum of the broad fluorescence band of the dimer in aqueous solution did not depend on the concentration and located at $605 \mathrm{~nm}$. The smaller Stokes-shift in the film suggests that the geometrical relaxation of the dimer in

40 the excited state is restricted in the solid state. The continuous redshift of the emission of the dimer suggests that the energy migration to the stable dimer site occurs in the aggregate and the number and/or the property of the stable site are dependent on the size of the aggregate. In addition, as will be shown in Fig. 6, the ${ }_{45}$ segregation of PeryAm aggregates in the PVA film was observed under high concentration. Hence, inhomogeneity of aggregates could be attributed also to the gradual redshift of the emission in the PVA film.
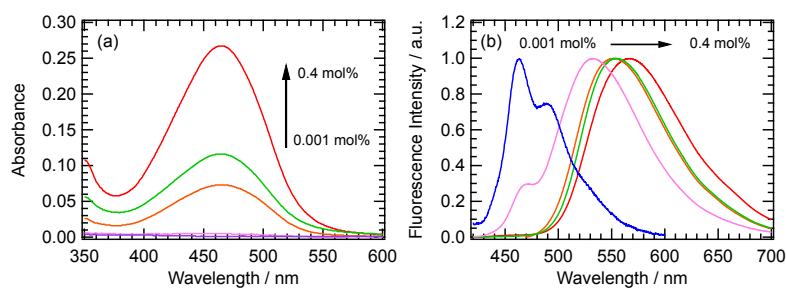

$50 \quad$ Fig. 3 Absorption (a) and fluorescence (b) spectra of PeryAm in PVA films at $0.001,0.01,0.1,0.2$ and $0.4 \mathrm{~mol} \%$. The excitation wavelength is $400 \mathrm{~nm}$.

To explore the contribution of the energy migration in the gradual redshift, steady-state fluorescence anisotropy $(r)$ was 55 measured. Details of the excitation anisotropy spectra were plotted as a function of concentration in ESI. The $r$ value under the excitation in shorter wavelength region was small, while larger $r$ values were observed by the excitation in longer wavelength region of the absorption band (see ESI). In Fig. 4, we 60 show the $r$ values monitored for the fluorescence at 530 and 650 $\mathrm{nm}$ as a function of PeryAm concentration in order to simply compare the efficiency of the energy migration in the aggregates depending on the monitoring wavelength as a function of the concentration. In this plot, the $r$ values were averaged in the ${ }_{65}$ range of 428 to $498 \mathrm{~nm}$ in the fluorescence excitation spectra. In the dilute condition at $0.01 \mathrm{~mol} \%$, the $r$ values were respectively $0.39(530 \mathrm{~nm})$ and $0.35(650 \mathrm{~nm})$, indicating that the direction of transition dipoles is almost the same between the absorption and emission transitions. With an increase in the concentration, both 70 the $r$ values decrease but the decrement of the $r$ value at $650 \mathrm{~nm}$ is more strongly dependent on the concentration. This result strongly suggests that the energy migration into more stable sites is pronounced with increasing concentration.

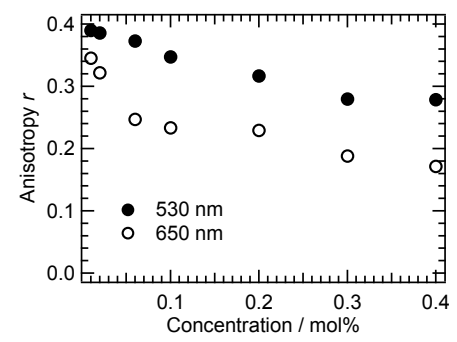

75 Fig. 4 Fluorescence anisotropy of PeryAm in PVA films. The monitoring wavelength is 530 (closed circles) and $650 \mathrm{~nm}$ (opened circles). The $r$ values were averaged for the excitation in $430-500 \mathrm{~nm}$ region.

To more directly elucidate the energy migration, we measured fluorescence time profiles of PeryAm in PVA films excited at ${ }_{80} 400 \mathrm{~nm}$. The monomer and dimer of PeryAm in the film were excited at $400 \mathrm{~nm}$ and the population ratio could be estimated to be $1: 3$ on the basis of the association constant in aqueous solution. The ratio of the excited species may be inclined to the dimer to more extent, because the absorption coefficient of the dimer at ${ }_{85} 400 \mathrm{~nm}$ is still large (close to $10^{-4} \mathrm{M}^{-1} \mathrm{~cm}^{-1}$ ) as was shown in Fig.1.

Fig. 5 shows time profiles for the film with 0.4 mol\% PeryAm, monitored at 470 and $650 \mathrm{~nm}$. Fluorescence at $470 \mathrm{~nm}$ (Fig. 5(a)) is mainly attributed to the emission from the monomer species as 90 shown in the absorption and fluorescence spectra in Fig. 3. Time 
profile at $470 \mathrm{~nm}$ was reproduced by a triple exponential function with time constants of $9 \mathrm{ps}, 450 \mathrm{ps}$, and 3.49 ns and the major components of the decay were 9 and 450 ps. These behaviors strongly suggest that the monomer species underwent the rapid 5 energy transfer to the dimer site in aggregates. On the other hand, time profile at $650 \mathrm{~nm}$ shows a fast rise component with 10-ps time constant and decay components of 3.8 and $12 \mathrm{~ns}$. The time constant of the rise component is almost the same with that observed in the decay at $470 \mathrm{~nm}$. These behaviors strongly

10 support the efficient energy migration leading to the formation of the excited state of the stable dimer species. It should be noted that aggregates with different sizes are segregated in the film with $0.4 \mathrm{~mol} \%$ PeryAm. In addition, the fluorescence time profiles were measured for rather wide spatial region of the film. Hence, 15 the fluorescence time profile is composed of those for many kinds of aggregates and multiple decay components may be attributed to these contributions. Actually, the multiple emissive species coexisting in the films also may contribute to the continuous redshift of the fluorescence shown in Fig. 3.
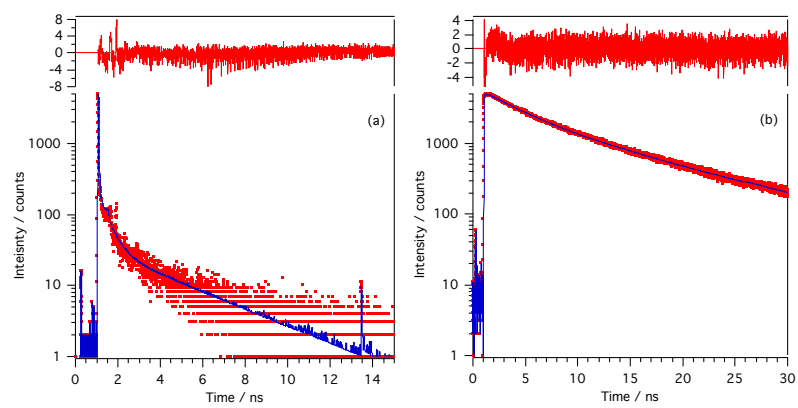

Fig. 5 Fluorescence time profiles of $0.4 \mathrm{~mol} \%$ PerAm in PVA film excited at $400 \mathrm{~nm}$ and monitored at 470 (a) and $650 \mathrm{~nm}$ (b).

\section{Fluorescence images of PeryAm in PVA thin films under the} ${ }_{25}$ optical microscope

In order to acquire the information on the relation between fluorescence properties and the morphology of aggregates, we measured fluorescence microscope images of PeryAm in PVA films excited the UV light in Fig. 6. In the film with $0.01 \mathrm{~mol} \%$ 30 (Fig. 6a), fluorescence spots with blue and green color are observed. These emissions are respectively attributable to the monomer and dimer of PeryAm, of which results are consistent with the ensemble fluorescence spectrum shown in Fig. 3. At the concentration of $0.02 \mathrm{~mol} \%$, fluorescence image shows green

35 color. Because the fluorescence intensity is not uniform in all the areas of the image and localizes in many tiny spots, the emission is mainly due to small aggregates at this concentration. Over 0.2 mol\%, the background emission turns from blue-green to yellowish green. In addition, bright spots and several types of the 40 emission with green, yellow and orange, are observed. The yellow fluorescence is detected in large aggregates with the size $\geq$ $10 \mu \mathrm{m}$. Because this yellow spot was independent of the polarization of the detection, the structure of the aggregate is in the amorphous state. The orange-colored emission is quite weak 45 and the spot looks like dark shadow (Inset of Fig. 6d). This is probably due to the low fluorescence quantum yield of the orange-colored emission, of which value was estimated to be less than 0.1 . The image of the aggregate with orange emission was dependent on the polarization, suggesting that this aggregate has ${ }_{50}$ crystal properties. The fluorescence spectra are also different in yellow- and orange-colored species under the microscope as shown in Fig.7.

From the comparison of the images between 0.2 and $0.4 \mathrm{~mol} \%$, one can find that the number of yellow-colored aggregates in the ${ }_{55}$ film with the $0.4-\mathrm{mol} \%$ PeryAm is much larger than that of orange one. As mentioned in the previous section, the structure of the yellow-colored aggregate is in the amorphous state, while the orange-colored emission was suggested to be in the crystalline phase. The larger number of the yellow-colored aggregate in the 60 film with the $0.4-\mathrm{mol} \%$ PeryAm strongly suggests that the change of the fluorescence color is not simply due to the crystal formation. 


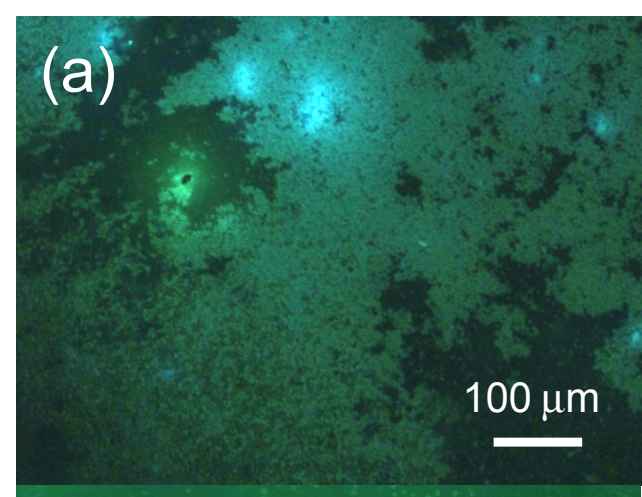

(b)

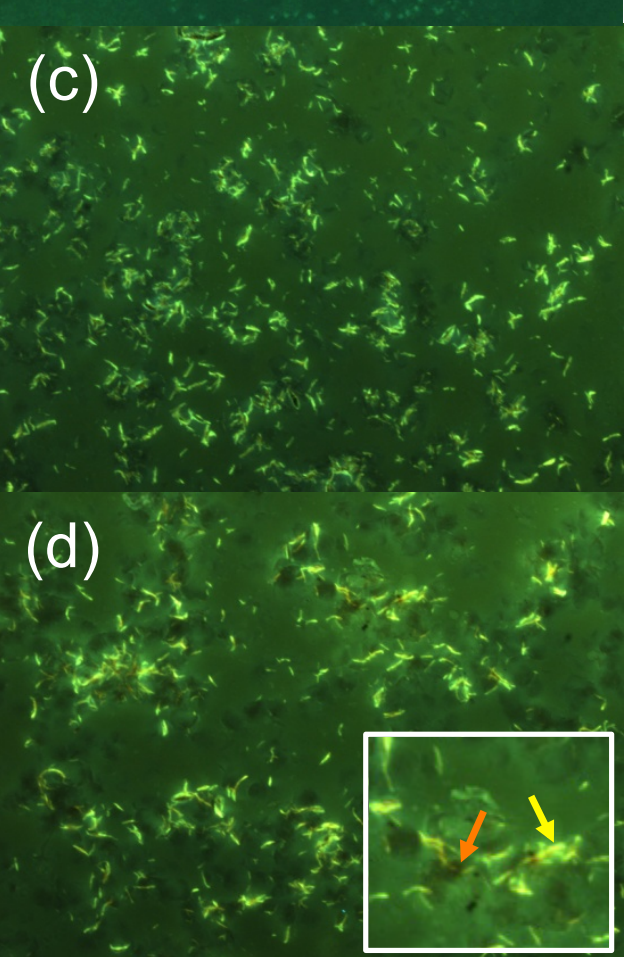

Fig. 6 Fluorescence microscope images of PeryAm in PVA films; (a) 0.01 , (b) 0.02 , (c) 0.2 and (d) $0.4 \mathrm{~mol} \%$. Inset of (d) was an image expanded in scale, showing yellow- and orange-colored aggregates.

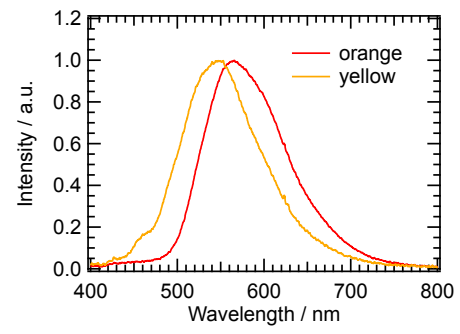

Fig. 7 Fluorescence spectra of PeryAm aggregates under the optical microscope.

\section{Dynamic change of emission color of aggregates under the} steady-state irradiation

To elucidate the dynamics of the aggregation and its relation to the change of the emission color, we measured the temporal evolution of the fluorescence image of the $0.3-\mathrm{mol} \%$ PeryAm in 15 PVA film under the steady-state light irradiation in the wavelength $<420 \mathrm{~nm}$ (Fig. 8). The green emission was observed around the yellow-colored aggregate just after the photoirradiation. Under the continuous irradiation, parts of the green emission (indicated by the while circle) changed its color 20 into yellow, as shown in the red arrows in Fig. 8. The number of the yellow-colored aggregate increased with elapse of irradiation time.

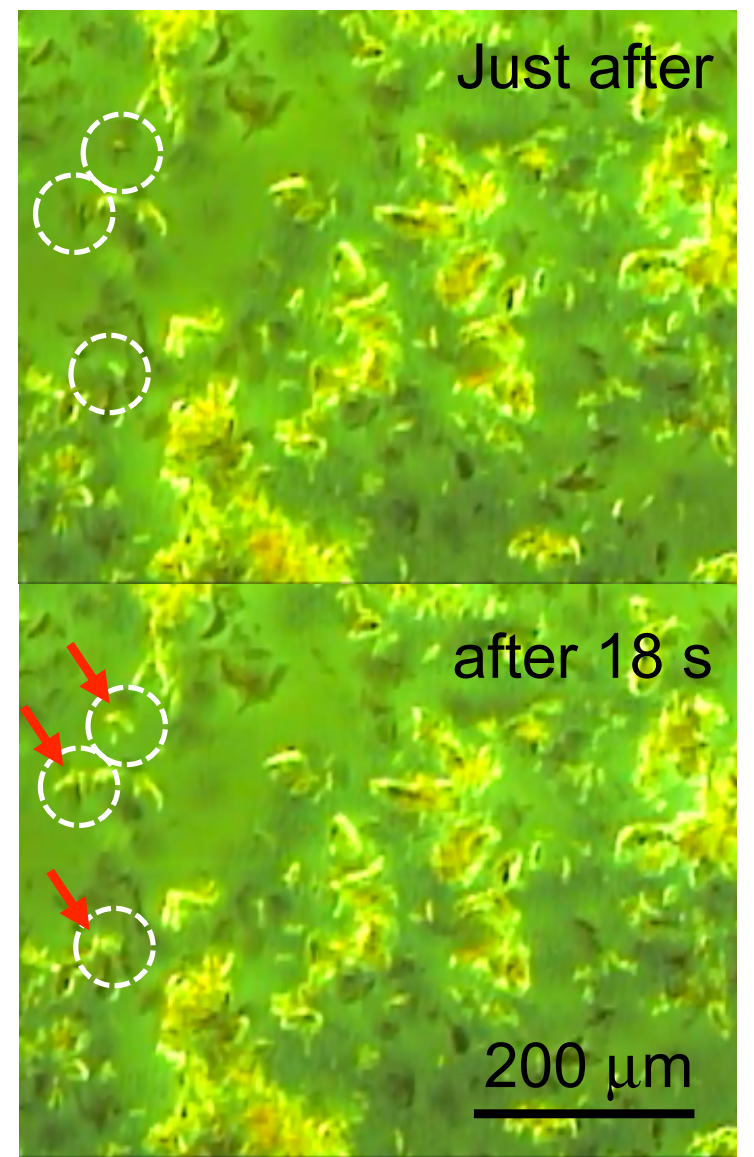

Fig. 8 Fluorescence microscope image of $0.3 \mathrm{~mol} \%$ PeryAm in PVA yellow-colored aggregates appearing by the photoirradiation. 
As shown in Figs. 3 and 6, the green-colored emission was mainly observed in the PVA film with low concentration of PeryAm. On the other hand, the yellow-colored emission was dominant in the film with the concentration $>0.2 \mathrm{~mol} \%$. In 5 addition, the yellow-colored emission was observed in rather large aggregates as was shown in Fig. 6. Fig. 8 shows that the change of the color from green to yellow is accompanied with the increase in the size of aggregates. This result directly indicates that the color of the emission is dependent on the size of 10 aggregates, which is consistent with the results in Figs. 3 and 6. In addition, the result in Fig. 8 shows that the color of the emission of PeryAm aggregates can be switched by the light irradiation, probably by the photothermal effect leading to the fusion of small aggregates.

\section{Assembling process of PeryAm in PVA films}

On the basis of the above results, the concentration-dependent fluorescence spectral change could be summarized in Fig. 9. The fluorescence color at $0.01 \mathrm{~mol} \%$ showed blue-green as was shown in Fig. 6a. These are mainly attributed to the monomer and 20 the dimer. At $0.02 \mathrm{~mol} \%$ where the segregation was observed in Fig. $6 \mathrm{~b}$, the color evolved into green-yellow. With further increase in the concentration, the size of the aggregate increased and the color of the fluorescence changed into yellow. The size of the aggregates was estimated to be ca. $10 \mu \mathrm{m}$ at $0.06 \mathrm{~mol} \%$. The 25 size increases with an increase in the concentration and it was ca. $30 \mu \mathrm{m}$ at $0.4 \mathrm{~mol} \%$ while keeping the yellow color. Because the yellow-colored fluorescence was independent of the polarization, it was suggested that the morphology was in the amorphous state. The small aggregate with orange-colored fluorescence appeared 30 at the concentration larger than $0.2 \mathrm{~mol} \%$. The polarizationdependent property of the orange emission suggested that the aggregate was in the crystalline phase. By comparing the present fluorescence spectrum with that of the crystal powder, ${ }^{12}$ it could be deduced that crystallization partially occurred in the films 35 upon increasing local concentration during solvent evaporation. Because the orange-colored emission was not detected in the concentration lower than $0.2 \mathrm{~mol} \%$, it could be concluded that the amorphous phase turned into the crystalline phase. Actually, as was shown in Figs. 6 and 8, the orange colored region was 40 located in the yellow-colored aggregate.

The characteristic fluorescence properties have been widely reported for organic nanoparticles. ${ }^{19-22}$ Kasai et al. reported that the optical properties of perylene nanoparticles prepared by the reprecipitation method. ${ }^{19,20}$ The fluorescence spectra of the 45 nanoparticles differ from that of the bulk crystals. They proposed the lattice softening in nanoparticles as a cause of the sizedependent optical properties. ${ }^{21,22}$ Such change of the properties of small particles allowing the geometrical relaxation may contributes to the present gradual shift of the emission and time so profiles of the fluorescence to some extents.

In general, the organic molecules with bulky substituent and highly conformational freedom easily form the amorphous state. PeryAm has ammonium group which is probably affected by conformation freedom. PeryAm is preferentially formed of 55 amorphous phase with yellow emission rather than crystal phase with orange emission. These findings suggest that the aggregation process is strongly dependent not only on the dispersion concentration of PeryAm, but also on the speed of the solvent evaporation during the casting process.

60

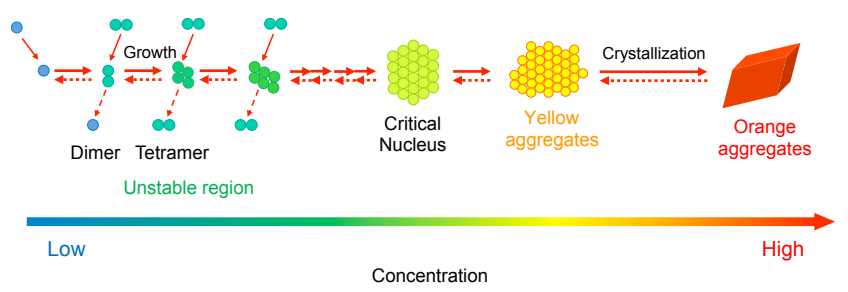

Fig. 9 Schematic representation of assembling process of PeryAm.

\section{Conclusions}

${ }_{65}$ We have investigated properties and evolution of PeryAm aggregates in PVA matrices based on the fluorescence properties. The fluorescence lifetime and anisotropy depending on the monitoring wavelength indicated that different emissive states, depending on the concentration, were produced in the PVA film. 70 Aggregates with the yellow-colored emission (in the amorphous phase) were produced prior to the appearance of the aggregates with orange-colored emission in the crystalline phase. The fluorescence image showing that the small part of the yellowcolored emission turned into orange-colored spot indicated that 75 the part of the amorphous area gradually turned into the crystalline phase.

The steady-state light irradiation induced the change of the emission color accompanied with the fusion of the small aggregates. This result suggests that the photo-thermal evolution 80 of the emission color in the aggregate is attainable by the photoirradiation.

\section{Acknowledgements}

The authors thank Prof. H. Usami (Shinshu University) for NMR measurement, Prof. K. Tanaka (Kyushu University) for the 85 elementary microanalysis, Prof. N. Tanaka (Shinshu University) for MS measurements. This work partly supported by Grant-inAid for Young Scientist (B) (No. 21750021) and for Scientific Research (C) (No. 26410009) from JSPS. FI was also supported by Iketani Science and Technology Foundation, and by the JGC${ }_{90}$ S Scholarship Foundation. HM and FI acknowledge the financial support of a Grant-in-Aid for Scientific Research on Innovative Areas "Photosynergetics" (No. 26107002 and 15H01081) from MEXT, Japan.

\section{Notes and references}

$95{ }^{a}$ Department of Chemistry, Institute of Education, Shinshu University, 6ro, Nishinagano, Nagano 380-8455, Japan. Fax: +81-26-238-4114; Tel: +81-26-238-4114; E-mail: fito@shinshu-u.ac.jp;

${ }^{b}$ Division of Frontier Materials Science, Graduate School of Engineering Science, Osaka University, Toyonaka, Osaka 560-8531, Japan.

$100 \dagger$ Electronic Supplementary Information (ESI) available: estimation of monomer-dimer equiribrium, fluorescence time profiles. See DOI: $10.1039 / \mathrm{b} 000000 \mathrm{x} /$

1 F. J. M. Hoeben, P. Jonkheijm, E. W. Meijer and A. Schenning, Chem. Rev., 2005, 105, 1491.

1052 M. Schwoerer and H. C. Wolf, Organic Molecular Solids, WileyVCH, Weinheim, Germany, 2007. 
3 F. Wurthner, Z. J. Chen, V. Dehm and V. Stepanenko, Chem. Commun., 2006, 1188.

4 J. M. Lim, P. Kim, M.-C. Yoon, J. Sung, V. Dehm, Z. Chen, F. Wuerthner and D. Kim, Chemical Science, 2013, 4, 388.

5 H. Tachikawa and L. R. Faulkner, Chem. Phys. Lett., 1976, 39, 436.

6 J. C. D. Verhagen, M. vanZandvoort, J. M. Vroom, L. B. A. Johansson and G. vanGinkel, J. Phys. Chem. B, 1997, 101, 10568.

7 C. Spies and R. Gehrke, J. Phys. Chem. A, 2002, 106, 5348.

8 Y. Amao and I. Okura, Bull. Chem. Soc. Jpn., 2002, 75, 389.

109 B. R. Crenshaw and C. Weder, Adv. Mater., 2005, 17, 1471.

10 T. T. Vu, M. Dvorko, E. Y. Schmidt, J. F. Audibert, P. Retailleau, B. A. Trofimov, R. B. Pansu, G. Clavier and R. Meallet-Renault, J. Phys. Chem. C, 2013, 117, 5373.

11 F. Ito, T. Kakiuchi, T. Sakano and T. Nagamura, Phys. Chem. Chem.

15 Phys., 2010, 12, 10923.

12 F. Ito, Y. Ugachi and T. Sasaki, Chem. Lett., 2012, 41, 282.

13 G. V. Zakharova, A. R. Kombaev and K. Chibisov, High Energ. Chem., 2004, 38, 180.

14 S. Sreeja, S. Sreedhanya, N. Smijesh, R. Philip and C. I. Muneera, J. 20 Mater. Chem. C, 2013, 1, 3851.

15 F. Ito, Y. Kogasaka and K. Yamamoto, J. Phys. Chem. B, 2013, 117, 3675 .

16 J. R. Lakowicz, Principles of Fluorescence Spectroscopy, Springer, Singapore, 2006.

2517 Y. Nagasawa, T. Itoh, M. Yasuda, Y. Ishibashi, S. Ito and H. Miyasaka, J. Phys. Chem. B, 2008, 112, 15758.

18 J. B. Birks, Photophysics of Aromatic Molecules, Wiley, London, 1970.

19 H. Kasai, H. S. Nalwa, H. Oikawa, S. Okada, H. Matsuda, N. Minami,

30 A. Kakuta, K. Ono, A. Mukoh and H. Nakanishi, Jpn. J. Appl. Phys. Part 2 - Lett., 1992, 31, L1132.

20 H. Kasai, H. Kamatani, Y. Yoshikawa, S. Okada, H. Oikawa, A. Watanabe, O. Itoh and H. Nakanishi, Chem. Lett., 1997, 1181.

21 T. Asahi, T. Sugiyama and H. Masuhara, Acc. Chem. Res., 2008, 41, 1790 .

22 T. Asahi, H. Matsune, K. Yamashita, H. Masuhara, H. Kasai and H. Nakanishi, Pol. J. Chem., 2008, 82, 687.

Graphical Abstract

40 Size-dependent fluorescent properties and evolution of emission in molecular aggregates of a perylene ammonium derivative in polymer matrices were investigated by steady-state and timeresolved spectroscopic methods.

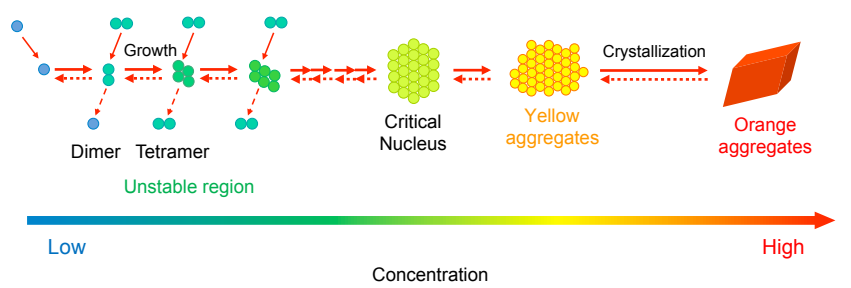

